\title{
GPPS-TC-2019-0048
}

\section{PLANAR LIF/MIE RATIO DROPLET SIZING USING STRUCTURED LASER SHEET IMAGING AT ELEVATED AMBIENT PRESSURES}

\author{
Andrew Corber \\ National Research Council of \\ Canada \\ Andrew.Corber@nrc.ca \\ Ottawa, Ontario, Canada
}

\author{
Wajid A. Chishty \\ National Research Council of \\ Canada \\ Wajid.Chishty@nrc.ca \\ Ottawa, Ontario, Canada
}

\author{
Patrizio Vena \\ National Research Council of \\ Canada \\ Patrizio.Vena@nrc.ca \\ Ottawa, Ontario, Canada
}

\begin{abstract}
LIF/Mie ratio-metric imaging was used to characterize sprays produced by a simple hollow-cone pressure atomizer, operating under elevated ambient pressures up to $10 \mathrm{~atm}$. A structured laser sheet was used as the source of illumination to suppress the multiple scattered light, generating images that are free of the artefacts typically found in conventional laser sheet images. The resulting LIF/Mie ratio-metric images were calibrated using Phase Doppler anemometry to generate axial planar maps of the spray's Sauter-mean diameter (SMD). This calibration methodology was applied over a range of ambient pressures and liquid flows to assess the robustness of the structured LIF/Mie ratio-metric imaging as a droplet sizing technique. The test fluids consisted of conventional and alternative jet fuels as well as nozzle calibration fluid. Results presented in the paper indicate both the effectiveness and certain limitations of the technique.
\end{abstract}

\section{INTRODUCTION}

Atomization of liquid fuels plays an important role in gas turbine combustion as its quality has a direct consequence on combustion efficiency and emissions of pollutants. As such, spray characterization is an essential step in the development of gas turbine combustors and fuel injectors, requiring reliable diagnostic techniques.

To fully characterize a spray large amounts of information are needed. Macro features, including overall spray dimensions such as width and cone angle are typically determined by photographic flow visualization. Micro features, such as droplet size, spatial distribution and velocity, require the application of more sophisticated diagnostics. Non-intrusive, laser based measurement tools, are widely used for this purpose and can provide the experimenter with substantial data about the spray.

The current state-of-the-art technique for measuring the size of droplets in a spray is the phase Doppler anemometer
(PDA), which is able to simultaneously measure both droplet size and velocity at a rate in excess of $20 \mathrm{kHz}$. PDA is a point based technique, requiring a large number of measurements be made at different locations over the entire spray to completely map the spatial distribution of droplet size and velocity. This is time consuming and consequently expensive given the high cost of testing at gas turbine representative pressures. Planar laser-based measurement methods, such as Laser Induced Fluorescence (LIF)/Mie ratio-metric imaging, have the potential to collect much larger amounts of data within considerably shorter time period compared to PDA. Unfortunately, such imaging techniques have serious drawbacks and limitations.

As the number of particles in the spray increases as a result of higher liquid flow rates and improved atomization, measurements become more and more challenging due to the laser light attenuating as it travels through the spray. This is exacerbated further by virtue of the light being scattered multiple times as it travels from its source to the detection plane, producing erroneous signals in the process and making features on the interior of the spray difficult to measure. These phenomena explain why many of the conventional images of hollow cone sprays found the literature have visible cores at low flows and pressures, but appear to be solid cones at high power conditions. An example of this is demonstrated in Figure 1, which shows how the centre of a hollow cone spray is visible at lower ambient pressure, but is obscured at higher pressures by the multiple scattering caused by the dense field of droplets.

A method for suppressing the multiple scattering effects has been demonstrated by Berrocal and co-workers (Berrocal et al. 2008). They used a laser sheet with a modulated intensity profile (i.e. sinusoidal intensity pattern) and post processing algorithms to generate images of a spray that are free of multiple scattering artefacts. Using PDA, (Mishra et al. 2015) showed that a LIF/Mie ratio-metric image collected with conventional laser sheet imaging could not be calibrated because the resulting intensity ratios in the image did not 
correspond to a singular droplet Sauter-mean diameter (SMD). By incorporating a Structured Laser Illumination Planar Imaging (SLIPI) strategy into the measurement system, they were able to apply LIF/Mie imaging to capture images that could be calibrated. This work represented a significant advancement in spray diagnostics, as it enabled the measurement of a SMD map in a few seconds: a task that would take hours with conventional methods. However, they only presented data at one axial plane for one image. As a result, questions still remain in regard to how widely the calibration can be applied, which is the focus of this paper.

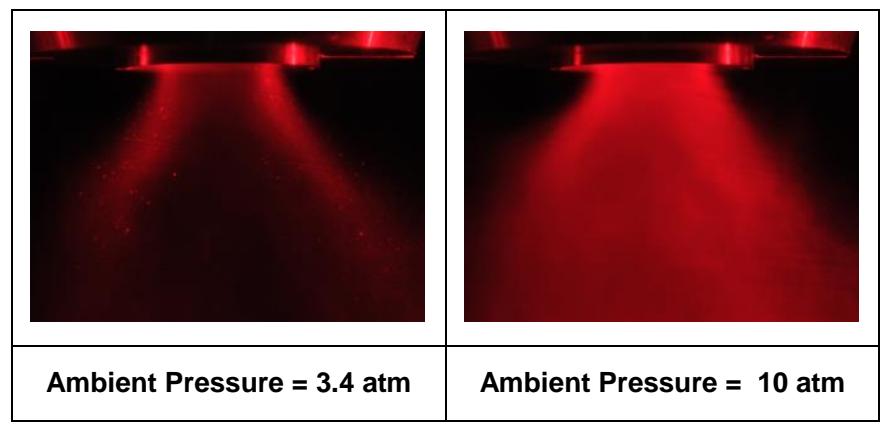

Figure 1: Laser sheet images of a hollow cone fuel spray at low and high pressure.

\section{METHODOLOGY}

\section{Test Facility}

All testing was completed in the National Research Council of Canada's (NRC) High Pressure Spray Facility (HPSF) in Ottawa, Canada. This test rig consists of a pressure vessel with large optical access that can be used to study sprays over ambient pressure conditions ranging from near atmospheric to 20 bar(a). The piping and instrumentation diagram used for all tests is shown in Figure 2. Process control is achieved via Lookout software, while operating conditions are monitored and recorded using LabView software. Further details on the operation and capacity of this facility can be found in (Corber 2007).

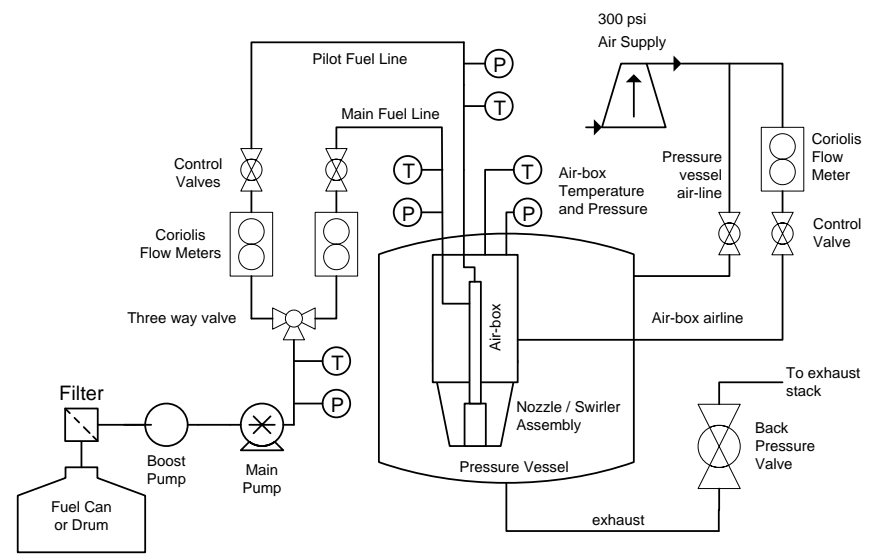

Figure 2: Piping and Instrumentation Diagram

\section{Optical Diagnostics}

In order to assess the capability of the SLIPI based LIF/Mie ratio-metric method to provide robust and quantitative droplet sizing data, experiments were carried out on sprays over a range liquid flows and ambient pressures using both conventional and alternative fuels as well as a calibration fluid as shown in Table 1. Conventional laser sheet images were collected to help visualize global spray features as well as to offer a comparison to the SLIPI images. PDA measurements were made at selected points in order to calibrate the SLIPI images and obtain SMD maps of the sprays.

The conventional laser sheet imaging instrumentation employed a 5W DPSS laser, manufactured by UltraLasers, as the light source. It operates at $532 \mathrm{~nm}$ and was equipped with sheet forming optics. A Cannon 5D Mark II digital SLR, equipped with a Canon $24-105$ f4.0 L-series lens, was used to capture the images. The ratio-metric imaging technique involves doping the fuel with a fluorescing dye, and illuminating the spray with a laser sheet. Two images of the spray are then collected simultaneously, with one of the cameras using a filter to collect the LIF signal, and the other the Mie signal. These two images are then combined to give the LIF/Mie ratio which is nominally proportional to SMD. A more detailed explanation of the technique can be found in (Mishra et al. 2015). The system used to capture LIF/Mie images uses the same laser as the conventional imaging that is then modulated using a universal grating based structured laser illumination planar imaging optics module manufactured by LaVision Inc. Two LaVision Imager ProX cameras controlled by a programmable timing unit are used to capture pictures of the spray. The system was set up to only view half the spray, which allowed for a greater vertical field of view, while still optimizing the resolution of the camera's chip. The configuration is shown in Figure 3.

For conventional drop sizing and velocity measurements, a commercial Phase Doppler Particle Analyzer (PDPA), manufactured by TSI Inc. was used in this study. The system consists of a TM250 transmitter, equipped with a beam expander and a 500mm lens. This results in a velocity range of $-225 \mathrm{~m} / \mathrm{s}$ to $1125 \mathrm{~m} / \mathrm{s}$. The receiver, model number RV2100, was orientated in the 30 degree forwardscatter configuration. Using this optical path, the PDPA can measure particle diameters from approximately $1-266 \mu \mathrm{m}$.

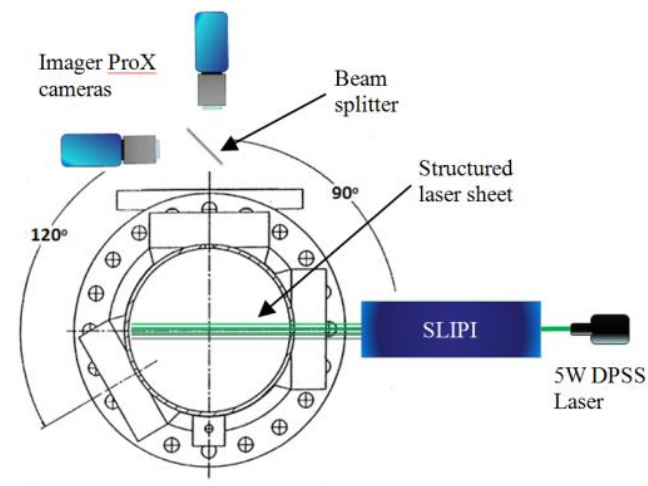

Figure 3: Experimental set up of LIF/Mie Ratiometric imaging system. Top View. 


\section{Fuel Nozzle}

All testing was conducting using an aero-engine derived fuel injector. A sketch of the nozzle is shown in Figure 4. For the results reported here, only the pilot fuel line was used, which is a simple-orifice type pressure atomizer. It has a flow number (FN) of $0.2 \mathrm{~g} / \mathrm{s} / \mathrm{kPa}^{1 / 2}$ and produces a hollow cone spray. For further details on the design and operation of the fuel injector see (Corber et al. 2018).

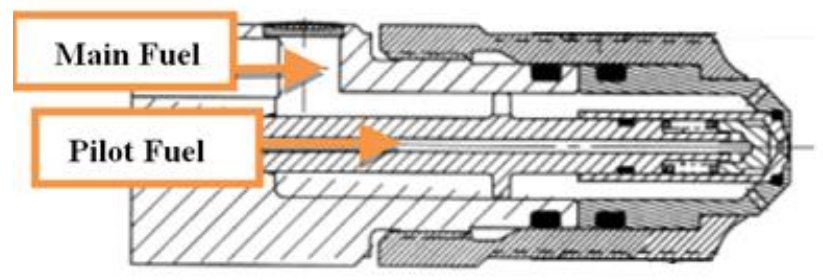

Figure 4: Simple-orifice pressure atomizer

\section{Test Liquids}

Three test liquids were employed in this study. A brief summary of their distinguishing features is shown in Table 1. These fluids were selected for their range of physical properties, as well as represent separate categories: calibration fluid, conventional jet fuel, and alternative jet fuel respectively.

Table 1 Test Fluid Properties

\begin{tabular}{|c|l|l|c|}
\hline Fuel & \multicolumn{1}{|c|}{ Composition } & \multicolumn{1}{|c|}{ Features } & $\begin{array}{c}\text { Viscosity } \\
\text { 25 C } \\
\text { [cSt] }\end{array}$ \\
\hline MIL & $\begin{array}{l}\text { Nozzle calibration } \\
\text { fluid }\end{array}$ & $\begin{array}{l}\text { Similar physical } \\
\text { properties to A-2. } \\
\text { Less flammable. }\end{array}$ & 1.2 \\
\hline C-3 & $\begin{array}{l}\text { "Average" or } \\
\text { "nominal" jet fuel } \\
\text { [Jet A] }\end{array}$ & $\begin{array}{l}\text { Average } \\
\text { properties. }\end{array}$ & 1.7 \\
\hline $\begin{array}{l}\text { 64 vol\% high } \\
\text { (A-3)/36 vol\% } \\
\text { farnesane }\end{array}$ & $\begin{array}{l}\text { Very high } \\
\text { viscosity jet fuel, } \\
\text { (viscosity spec } \\
\text { limit). }\end{array}$ & 2.3 \\
\hline
\end{tabular}

\section{Test Matrix}

The complete test matrix for the experimental campaign is shown in Table 2. It is derived from the test points provided by the National Jet Fuel Combustion Program (NJFCP), more details of which can be found in (Colket et. al. 2017). It has been expanded slightly to cover a wider range of flows than those used in previous work (Corber et al. 2018) so that the application of the SLIPI LIF/Mie method can be tested over a greater variety of sprays shapes, sizes, and droplet diameters. All three fluids were tested at these operating conditions.
Table 2 Test Matrix

\begin{tabular}{|c|c|c|c|c|}
\hline $\begin{array}{c}\text { Test } \\
\text { Point }\end{array}$ & $\begin{array}{c}\boldsymbol{P}_{\mathbf{3}} \\
{[\mathbf{a t m}]}\end{array}$ & $\begin{array}{c}\Delta \boldsymbol{P}_{\boldsymbol{f}} \\
{[\mathbf{k P a}]}\end{array}$ & $\begin{array}{c}\dot{\boldsymbol{m}}_{\boldsymbol{f}} \\
{[\mathbf{g} / \mathbf{s}]}\end{array}$ & $\begin{array}{c}\dot{\boldsymbol{m}}_{\boldsymbol{w}} \\
{[\mathbf{k g} / \mathbf{s}]}\end{array}$ \\
\hline $\mathbf{1}$ & 1.1 & 100 & 2 & 0.5 \\
\hline $\mathbf{2}$ & 1.1 & 225 & 3 & 0.5 \\
\hline $\mathbf{3}$ & 1.1 & 400 & 4 & 0.5 \\
\hline $\mathbf{4}$ & 1.1 & 625 & 5 & 0.5 \\
\hline $\mathbf{5}$ & 2.0 & 100 & 2 & 0.5 \\
\hline $\mathbf{6}$ & 2.0 & 225 & 3 & 0.5 \\
\hline $\mathbf{7}$ & 2.0 & 400 & 4 & 0.5 \\
\hline $\mathbf{8}$ & 2.0 & 625 & 5 & 0.5 \\
\hline $\mathbf{9}$ & 3.4 & 100 & 2 & 0.5 \\
\hline $\mathbf{1 0}$ & 3.4 & 225 & 3 & 0.5 \\
\hline $\mathbf{1 1}$ & 3.4 & 400 & 4 & 0.5 \\
\hline $\mathbf{1 2}$ & 3.4 & 625 & 5 & 0.5 \\
\hline $\mathbf{1 3}$ & 5.0 & 100 & 2 & 0.5 \\
\hline $\mathbf{1 4}$ & 5.0 & 225 & 3 & 0.5 \\
\hline $\mathbf{1 5}$ & 5.0 & 400 & 4 & 0.5 \\
\hline $\mathbf{1 6}$ & 5.0 & 625 & 5 & 0.5 \\
\hline $\mathbf{1 7}$ & 6.8 & 100 & 2 & 0.5 \\
\hline $\mathbf{1 8}$ & 6.8 & 225 & 3 & 0.5 \\
\hline $\mathbf{1 9}$ & 6.8 & 400 & 4 & 0.5 \\
\hline $\mathbf{2 0}$ & 6.8 & 625 & 5 & 0.5 \\
\hline $\mathbf{2 1}$ & 10.0 & 100 & 2 & 0.5 \\
\hline $\mathbf{2 2}$ & 10.0 & 225 & 3 & 0.5 \\
\hline $\mathbf{2 3}$ & 10.0 & 400 & 4 & 0.5 \\
\hline $\mathbf{2 4}$ & 10.0 & 625 & 5 & 0.5 \\
\hline
\end{tabular}

\section{RESULTS AND DISCUSSION}

Due to space limitations, the presented results will focus on a limited number of test cases. Specifically, these include data for the A-2 and C-3 fuels at a flow rate of $5 \mathrm{~g} / \mathrm{s}$, at a rig operating pressures of $3.4 \mathrm{~atm}$ and $10 \mathrm{~atm}$.

\section{Conventional Laser Sheet Imaging - Results}

A sample of the conventional laser sheet images collected is shown in Figures 5 and 6. These pictures offer qualitative details with regard to the spray's overall features such as cone angle and quality of atomization. Details with regard to the spray's interior structures, such as if the spray is a solid or hollow cone, cannot be determined from these images. The results demonstrate that the spray changes significantly with rig operating pressure. As the ambient pressure increases the spray's cone angle collapses, resulting in a much more narrow spray. However, at the operating conditions presented, very little difference is observed between the various fuels. 


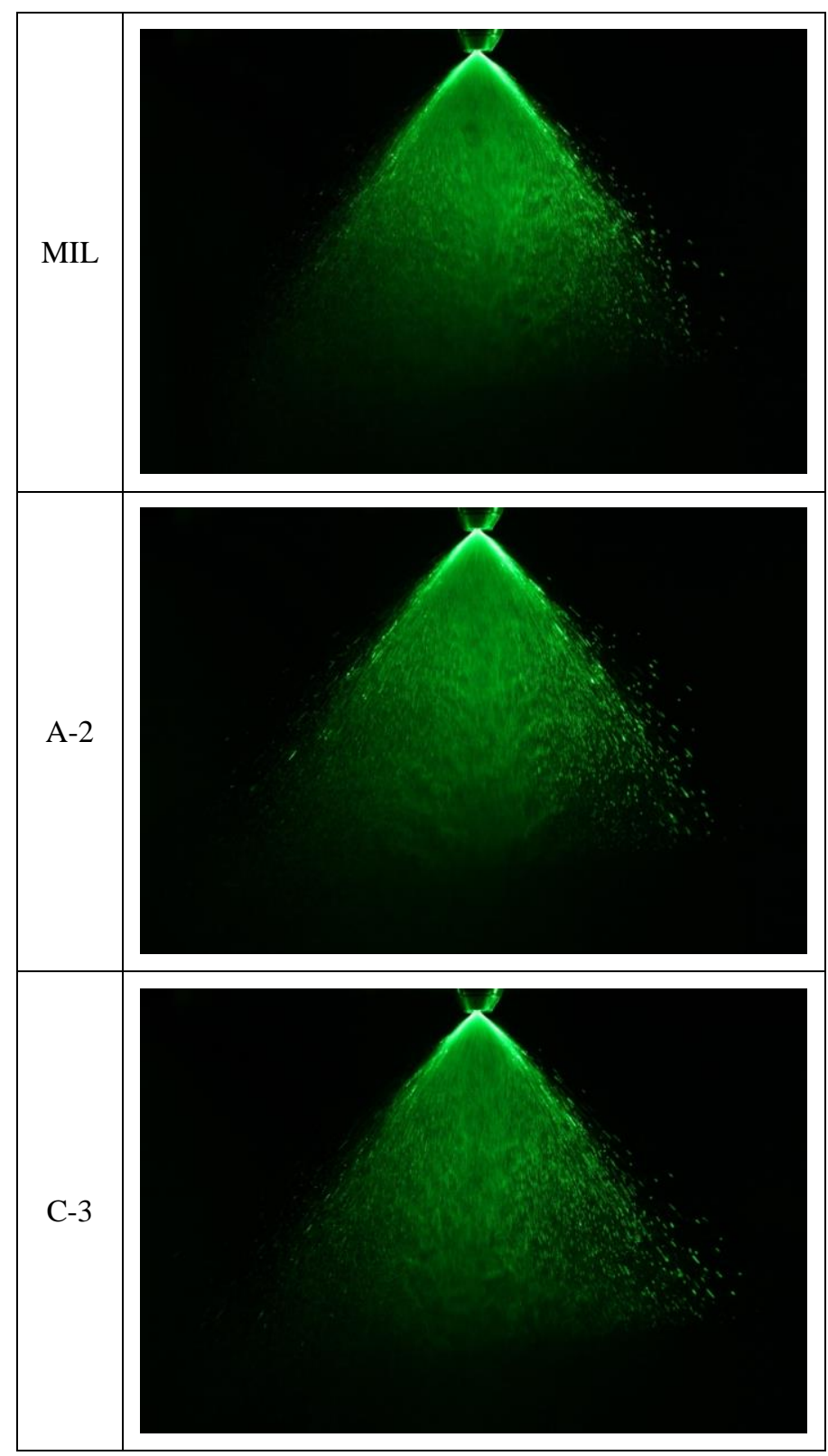

Figure 5: Conventional laser sheet images of MIL-C, A-2, C-3 fuel sprays. Fuel flow $5 \mathrm{~g} / \mathrm{s}$. $\mathrm{P}_{3}=3.4 \mathrm{~atm}$. Camera settings: $1 / 2000 s, \mathrm{f} / 6.3$, ISO -400 .

\section{Phase Doppler Anemometry Results}

Sample sets of PDPA data showing the radial distribution of droplet SMD for two test fuels at two axial locations downstream of the injector are given in Figures 7 and 8. During commissioning of the experimental setup, data points were taken across the entire width of the spray, and the measured droplet sizes were found to be symmetric about the centreline. Subsequent tests were then reduced to sampling on only half of the spray to lessen time and cost.

The PDPA was set up to collect 50,000 samples per point. A validation rate of $90 \%$ was achieved for the majority of cases, resulting in 45,000 valid droplet samples at each radial location.

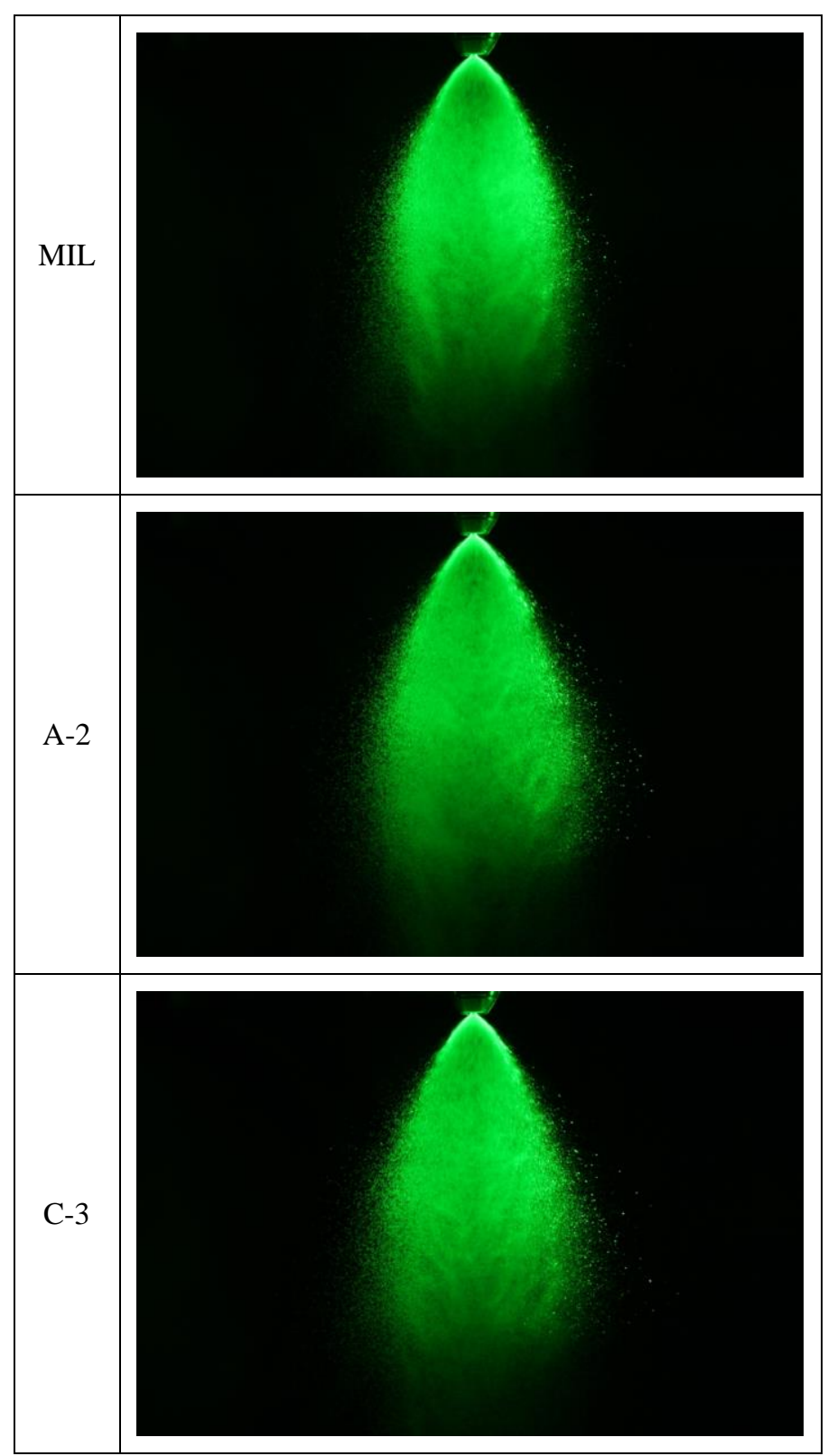

Figure 6: Conventional laser sheet images of MIL-C, A-2, C-3 fuel sprays. Fuel flow $5 \mathrm{~g} / \mathrm{s} . \mathrm{P}_{3}=10 \mathrm{~atm}$. Camera settings: $1 / 4000 s, \mathrm{f} / 6.3$, ISO-400.

The PDPA data qualitatively agree with the laser sheet images, indicating that a higher ambient pressure results in narrow sprays. It also indicates that the lower viscosity fuel, A-2, atomizes better (smaller droplets) than the higher viscosity C-3 fuel. This difference in atomization was however found to diminish at higher ambient pressures for the injector used in the investigation. Additionally, these plots indicate that the droplets size increases with increase in ambient pressure as well as with increase axial position. These results agree well those found in the literature (McDonell et al. 1994). 


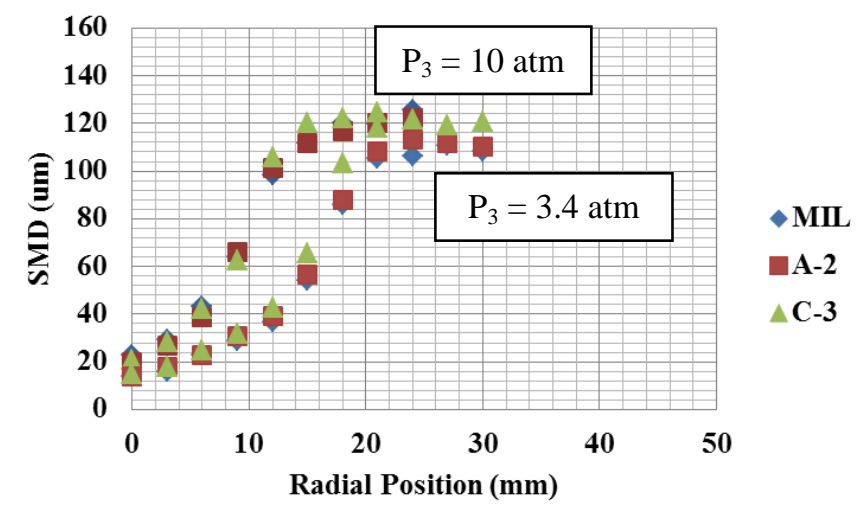

Figure 7: Droplet SMD measured by PDA at $25.4 \mathrm{~mm}$ from the nozzle face for MIL-C, A-2 and C-3. Fuel mass flow $5 \mathrm{~g} / \mathrm{s}$.

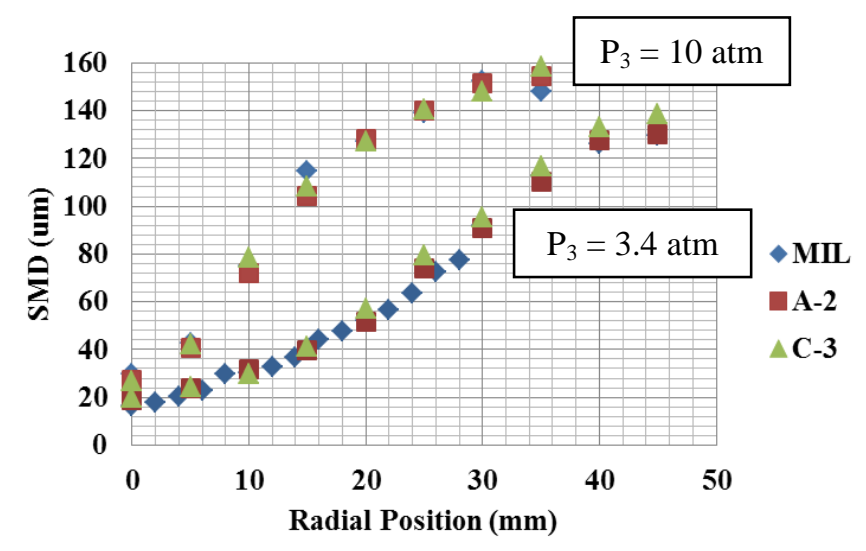

Figure 8: Droplet SMD measured by PDA at $50.4 \mathrm{~mm}$ from the nozzle face for MIL-C, A-2 and C-3. Fuel mass flow $5 \mathrm{~g} / \mathrm{s}$.

\section{SLIPI Ratio-metric Images}

Figure 8 (a) shows the typical resulting image generated by the SLIPI LIF/Mie ratio-technique. It is the result of the combination of $150 \mathrm{Mie}$ and 150 LIF SLIPI images. The normalized counts displayed in the figure are effectively the pixel-by-pixel ratio of the LIF and Mie images. Provided the fluorescing dye is evenly distributed throughout the liquid, the LIF signal is roughly proportional to the droplet volume. Since the Mie signal is roughly proportional to the droplet surface area, higher counts indicate areas in the spray with larger droplets (i.e. larger volume to surface area). Based on the work of (Mishra et al. 2015), the LIF/Mie ratio pixel count should correspond to a discreet SMD anywhere in the image. However, the count value itself is dependent on a number of parameters including, but not limited to, laser power, camera exposure time, particle number density, dye concentration, as well as the image processing methods. As a result, the absolute value of the pixel count is arbitrary, so in order to compare one ratio-metric image to another, pixel counts should be normalized. These normalized pixel counts cannot be used to determine droplet size directly, so in order to convert the image into a map of the droplet sizes an in-situ calibration is required to convert the intensity ratios to SMD's. The droplet size data collected by PDA was used to accomplish this, with the white dots in Figure 8 (b) showing the corresponding location of those measurements.

This technique does require a limited amount of image post processing. In order to reduce residual noise, a $20 \times 20$ pixel median filter, equivalent to $1 \times 1 \mathrm{~mm}$ in the flow, was applied to the images. Figure 8 (b) shows a resulting post processed image.

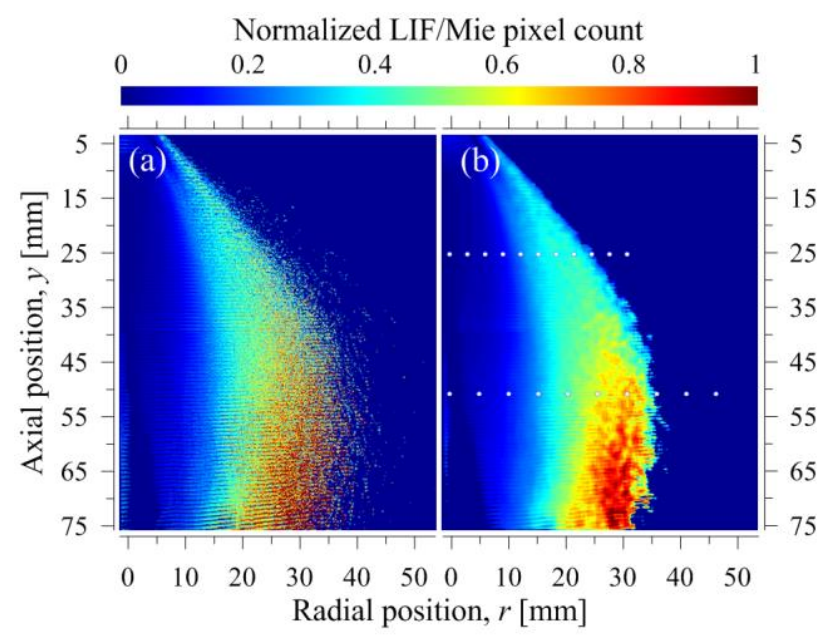

Figure 8: Uncalibrated SLIPI LIF/Mie ratio-metric image of C-3 spray with geometric scale. Fuel flow

$5 \mathrm{~g} / \mathrm{s}, P_{3}=10 \mathrm{~atm}$. (a) Raw image. (b) Post processed image with location of PDPA data.

PDA data was collected at two or more axial locations in the spray to determine the robustness of the calibration within a single image. This was also done to help determine if the location of the calibration measurements affects the SMD to pixel count calibration curve. To determine the consistency of the calibration across operating conditions and test fuels, calibration curves have been generated for MIL-C, A-2 and C-3 at two rig operating pressures for a liquid flow rate of $5 \mathrm{~g} / \mathrm{s}$. The results are shown in Figure 9.

Figure 9 shows the overall shape of the calibration curves to be consistent across all cases. At the 10 atm condition the calibration curves generated from the PDA data at $25.4 \mathrm{~mm}$ and $50.8 \mathrm{~mm}$ planes correlate closely, while for the 3.4 atm condition, the correlation between the two axial planes is not as high. Of all cases shown, the C-3 fuel at 10 atm offers the best results, with a nearly identical pixel count to SMD curve. There are several factors that help explain this. Based on the PDA data, the C-3 fuel at the 10 atm condition produces the spray with widest range of droplet sizes. As a result, this offers the largest calibration range, helping to reduce uncertainty. In addition, these larger droplets fluoresce and scatter the light with greater intensity. This leads to superior signal to noise ratios which also assists in improving the accuracy. 


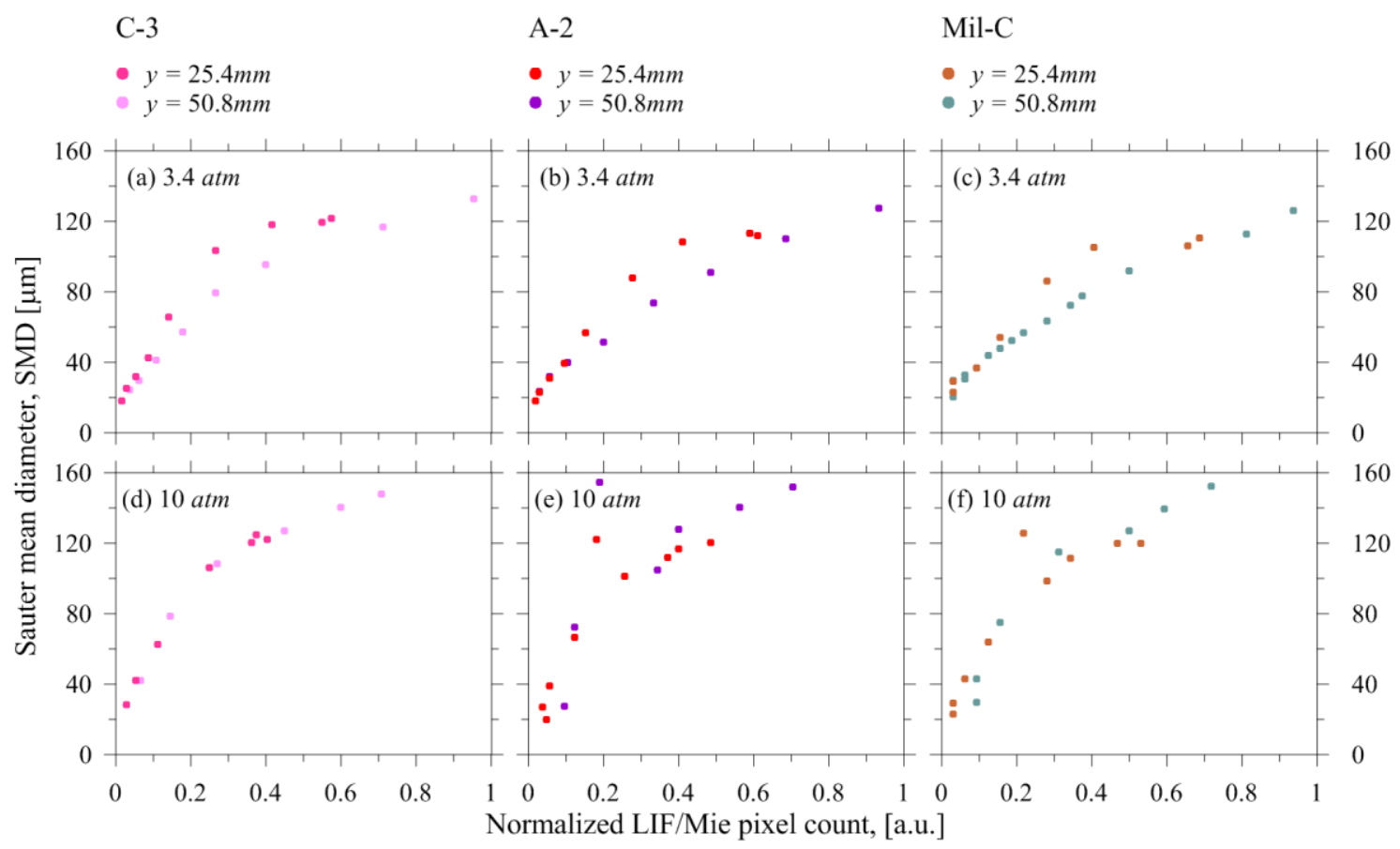

Figure 9: Calibration curves for C-3, A-2 and MIL-C LIF/Mie ratio-metric images at $P_{3}=3.4$ atm and 10 atm. Fuel mass flow $5 \mathrm{~g} / \mathrm{s}$.

In order to determine the exact mechanisms causing the difference between the upper and lower planes for the 3.4 atm condition (i.e. Figure 9 (a), 9 (b)) further investigation is required. A detailed examination into the sources of uncertainty, in conjunction with a statistical analysis for a large number of cases will need to be completed before conclusions can be drawn.

A second order exponential decay function was fit to relate pixel count to SMD in the LIF/Mie images. When applied to the image shown in Figure 8 (b), the result is a planar map of the droplet SMD's in the spray, and is shown in Figure 10. This plot is generated from a single calibration curve. It is observed to have the same overall trend as the PDPA data with larger droplets at the exterior of the spray, which increase in size as they travel downstream. Based on Figure 10, a single SLIPI LIF/Mie ratio-metric image can provide a spatially resolved droplet SMD's map. This is significant, since in order to produce the same chart, hundreds of PDA points and several hours of testing would be needed.

Figure 11 shows a comparison between a conventional laser sheet image, and an un-calibrated SLIPI ratio-metric image. While both offer details about the shape of the spray, there is significantly more information in the latter. The SLIPI demonstrates the hollow cone nature of the spray, as well as trends in droplet size. Even though a calibration may still be required in many applications to determine the exact SMD distribution, these results show a measurable gain in capability over conventional diagnostics.

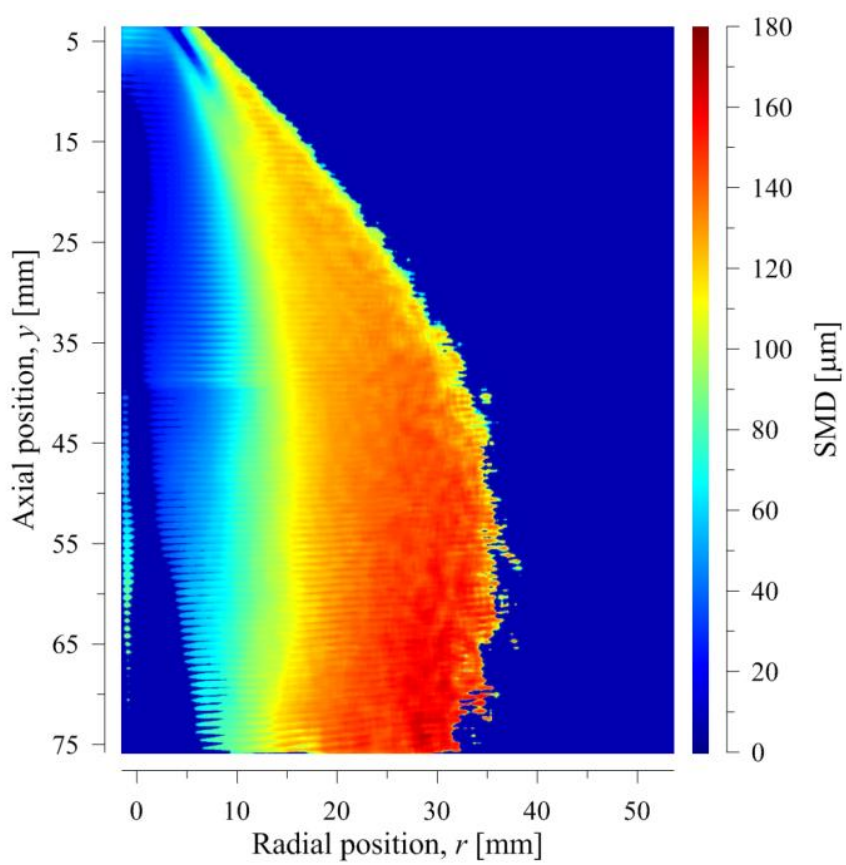
Figure 10: Planar SMD map (Calibrated SLIPI LIF/Mie ratio-metric image) of C-3 spray. Fuel flow $5 \mathrm{~g} / \mathrm{s}, P_{3}=10 \mathrm{~atm}$.




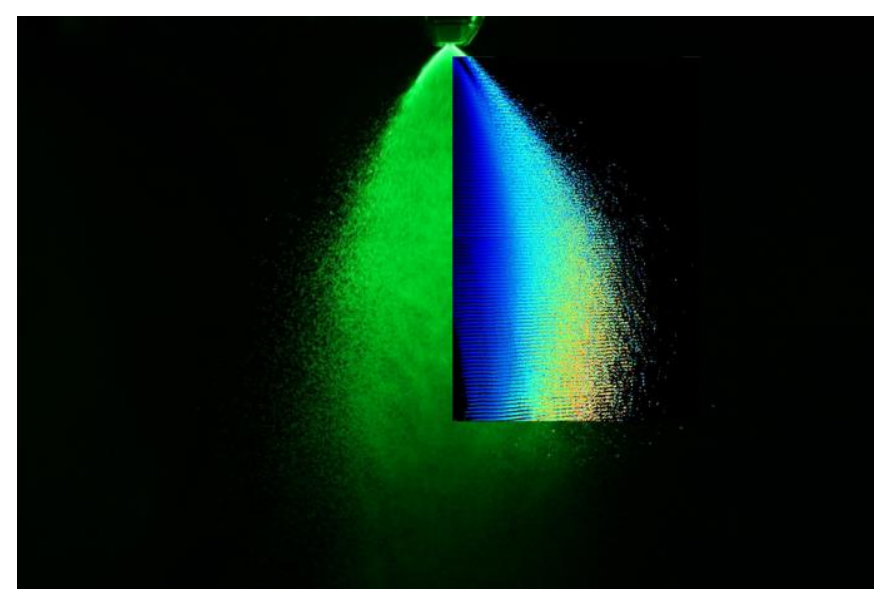

Figure 11: Un-calibrated SLIPI LIF/Mie ratio-metric image of C-3 spray overlaid on conventional laser sheet image. Fuel flow $5 \mathrm{~g} / \mathrm{s}, P_{3}=10 \mathrm{~atm}$.

\section{CONCLUSIONS}

An experimental investigation to assess the calibration of SLIPI LIF/Mie ratio-metric imaging has been presented. Tests were conducted using a nozzle calibration fluid, a conventional jet fuel and an alternative jet fuel, over a range of ambient pressures between 1.1 and 10 atm. PDA was used to measure the size of the droplets in the spray at two axial planes. The PDA data was then used to calibrate the LIF/Mie intensity ratios in the SLIPI images. The resulting calibration curves were then compared to determine their consistency across a single image, as well as between operating conditions and test fluids. This comparison showed the best agreement was achieved at higher ambient pressures, with the high viscosity fuel offering the best precision. This is due to the higher viscosity fuel spray having the widest range of droplet sizes, which not only gives a wider calibration curve, but also results in better signal to noise ratios in the final image. The relatively poor agreement between the calibration curves at the lower operating pressure indicates the need for a comprehensive uncertainty assessment to determine the robustness of this method. This incongruity also shows the importance of collecting PDA calibration data at locations in the spray that offer as large a range of droplet sizes as possible. In order to determine those locations, it is recommended that for an experimental campaign in which ratio-metric images are to be calibrated, the LIF/Mie images should be collected first. This will allow the experimenter to determine where the small and large particles exist so that the PDA data points can be collected strategically. The results shown here indicate that a single calibration curve may have the potential to be applied across multiple test points, provided the droplet size range is large enough, and that the signal to noise ratios in the images are sufficiently high. This is noteworthy since it will allow for large amounts of spatially resolved SMD data to be collected from a limited amount of PDA points, greatly reducing the time and cost of testing.

\section{NOMENCLATURE}

$\mathrm{P}_{3} \quad$ test section pressure, $\mathrm{kPa}$

$\Delta \mathrm{P}_{\mathrm{f}} \quad$ differential fuel pressure, $\mathrm{kPa}$

$\mathrm{m}_{\mathrm{f}} \quad$ mass flow of fuel, $\mathrm{g} / \mathrm{s}$

$\mathrm{m}_{\mathrm{w}} \quad$ mass flow of wash air, $\mathrm{kg} / \mathrm{s}$

CCD Charged Couple Device

FN Flow Number

HPSF High Pressure Spray Facility

NJFCP National Jet Fuel Combustion Program

NRC National Research Council

LIF Laser Induced Fluorescence

PDA Phase Doppler Anemometry

PDPA Phase Doppler Particle Analyzer

PTU Programmable Timing Unit

P\&ID Piping and Instrumentation Diagram

SLIPI Structured Laser Illumination Planar Imaging

SMD Sauter-Mean Diameter (D32), $\mu \mathrm{m}$

\section{REFERENCES}

Berrocal E., Kristensson E., Richter M., Linne M. and Aldén M. (2008), Application of structured illumination for multiple scattering suppression in planar laser imaging of dense sprays, Opt. Express, 16, 17870-17881.

Berrocal E., Kristensson E., Hottenbach P., Grünefeld G., and Aldén M., Quantitative laser imaging of a nonreacting diesel spray using SLIPI. ILASS Americas, 23rd Annual Conference on Liquid Atomization and Spray Systems, Ventura, CA, May 2011

Buschhagen T., Zhang R. Z., Bokhart A. J., Gejji R. M., Naikz S. V., Lucht R. P., Gore J. P., Sojka P. E., Slabaugh C. D., Meyer S. E. (2016). Effect of Aviation Fuel Type on Fuel Injection Conditions on Non-reacting Spray Characteristics of a Hybrid Airblast Fuel Injector. 54th AIAA Aerospace Sciences Meeting AIAA SciTech Forum, (AIAA 20161154).

Colket M. , Heyne J., Rumizen M., Gupta M., Edwards T., Roquemore W. M., Andac G., Boehm R., Lovett J., Williams R., Condevaux J., Turner D., Rizk N., Tishkoff J., Li C., Moder J., Friend D., Sankaran V. (2017). Overview of the National Jet Fuels Combustion Program. (AIAA 2017 $55: 4,1087-1104)$

Corber A. (2007). A Preliminary Investigation of Flow Scaling for Injector Characterization. ILASS Americas, 20th Annual Conference on Liquid Atomization and Spray Systems, Chicago, IL, May 2007.

Corber A., Rizk N., Chishty W. A. (2018). Experimental and Analytical Characterization of Alternative Aviation Fuel Sprays Under Realistic Operating Conditions. Proceedings of ASME Turbo Expo 2018, Oslo, Norway, GT2018-75574. 
Lefebrve A. H. and McDonell V. G (2017) Atomization and Sprays. CRC Press: Taylor \& Francis Group

McDonell V. G., Samuelsen G. S., Wang M. R., Hong, C. H. and Lai W. H. (1994). Interlaboratory comparison of phase Doppler measurements in a research simplex atomizer spray, Journal of Propulsion and Power, Vol. 10, No. 3 (1994), pp. 402-409.

McDonell V. G., Samuelsen G. S. (1995), An Experimental Data Base for the Computational Fluid Dynamics of Reacting and Nonreacting Methanol Sprays. ASME. J. Fluids Eng. 1995;117(1):145-153. doi:10.1115/1.2816804

Mishra, Y. M., Kristensson E., Berrocal E. (2015). Reliable LIF/Mie droplet sizing in sprays using structured laser illumination planar imaging. Optics Express, 22:44804492.

\section{ACKNOWLEDGMENTS}

The financial support for the experimental work comes from the Aeronautical Product Development Technologies program of National Research Council Canada and Transport Canada. The authors are grateful for the technical support from Mr. Daniel Arbique and Mr. Nicholas Charest of National Research Council Canada. The authors would also like to thank US AFRL for supplying the test fuels, as well as Edouard Berrocal and Elias Kristensson from Lund University for their assistance with the SLIPI set up and data reduction. 